\title{
1 The authenticity and quality of Rhodiola rosea products
}

2 Anthony Booker ${ }^{a}$, Banaz Jalil $^{\mathrm{a}}$, Debora Frommenwiler ${ }^{\mathrm{b}}$, Eike Reich ${ }^{\mathrm{b}}$, Lixiang Zhai ${ }^{\mathrm{a}, \mathrm{c}}$, Zarko Kulic ${ }^{\mathrm{d}}$, Michael

3 Heinrich $^{\mathrm{a}}$

$4{ }^{a}$ Centre for Pharmacognosy and Phytotherapy, UCL School of Pharmacy, Brunswick Square, London, UK,

$5{ }^{\mathrm{b}}$ CAMAG AG, 4132 Muttenz, Switzerland, ' Guangdong Pharmaceutical University, Guangzhou, China,

$6{ }^{\mathrm{d}}$ Preclinical Research, Dr. Willmar Schwabe GmbH \& Co. KG, Karlsruhe, Germany

7 Corresponding author: Michael Heinrich, Research Cluster Biodiversity and Medicines / Centre for

8 Pharmacognosy and Phytotherapy, UCL School of Pharmacy, Brunswick Square, London, UK. Tel: $+44(0) 20$

97753 5800, E-mail address: m.heinrich@ucl.ac.uk

Background: Rhodiola rosea L. root (Golden Root, Arctic Root) is a high-value herbal medicinal product, registered in the UK for the treatment of stress-induced fatigue, exhaustion and anxiety and used throughout Europe as a herbal medicinal product for similar indications. There are several Chinese species used in traditional Chinese medicine (TCM), including Rhodiola crenulata (Hook.f. \& Thomoson) that is believed to be a common adulterant in the R. rosea value chain. We investigate the phytochemistry of the different species and assess the potential of $R$. crenulata as an adulterant in the Rhodiola rosea value chain.

Aims: The project is embedded in a larger study aiming to investigate the diverse value chains that lead to the production of Rhodiola rosea as a herbal medicinal product. Here we focus on a comparison of the quality of the finished products and assess any phytochemical variation between products registered under the Traditional Herbal Medicine Products Directive (THMPD) and products obtained from the market without any registration (i.e. generally unlicensed supplements).

There are different species of Rhodiola on the market and the principal aim is to establish how these different species vary in their metabolite profile, how products are commercialised and whether there is potential for adulteration at the product manufacture stage.

Methods: Approximately 40 commercial products have been sourced from different suppliers. We analysed these samples using high performance thin layer chromatography (HPTLC), mass spectrometry (MS) and ${ }^{1} \mathrm{H}-$ NMR spectroscopy coupled with multi-variate analysis software following a method previously developed by our group for the analysis of turmeric products.

Results: The consistency of the products varies significantly. Approximately one fifth of commercial products that claimed to be Rhodiola rosea did not contain rosavin (one of the reference markers used to distinguish $R$. rosea from related species). Moreover some products appeared not to contain salidroside, another marker compound found in medicinal Rhodiola species. Approximately $80 \%$ of the remaining commercial products were lower in rosavin content than the registered products and appeared to be adulterated with other Rhodiola species.

Conclusions: The variation in phytochemical constituents present in Rhodiola products available to European buyers via the internet and other sources is a major cause for concern. Adulteration with different species, and other unknown adulterants, appears to be commonplace. Good quality systems and manufacturing practices, including those required under the THMPD, enable consumers to have confidence that products are authentic and meet a high specification for quality and safety. 


\section{Abbreviations}

43 AMIX

44 DMSO

45 GACP

46 GMP

$47 \quad \mathrm{HMP}$

$48 \quad$ HPTLC

49 HTP

$50 \quad$ LC

51 MS

$52 \quad$ NMR

53 PCA

$54 \quad \mathrm{PL}$

55

TCM

56 THR

57 THMPD

58

TMS
Analysis of mixtures

Dimethylsulphoxide

Good agricultural and collection practice

Good manufacturing practice

Herbal medicinal product

High performance thin layer chromatography

Hydroxytryptophan

Liquid chromatography

Mass spectrometry

Nuclear magnetic resonance

Principal component analysis

Product licence

Traditional Chinese medicine

Traditional herbal registration

Traditional Herbal Medicinal Products Directive

Tetramethylsilane

59

60

61

62

63

64

65

66

67

68

69 
Sedum roseum (L.) Scop., Crassulaceae, mainly known within the medicinal plant industry under its synonym Rhodiola rosea L., is a perennial flowering plant distributed throughout the northern hemisphere, particularly in circumpolar and high altitude regions of Europe and Asia, and to a lesser extent in North America (Brown, 2002).

Over 200 Rhodiola species have been documented, and many of these are used as medicine in Asia, including Rhodiola heterodonta (Hook.f. \& Thomson) Boriss, Rhodiola quadrifida (Pall.) Fisch \& Mey, Rhodiola semenovii (Regel \& Herder) Boriss, Rhodiola kirilowii (Regel) Maxim. and Rhodiola crenulata (Hook.f. \& Thomson) H.Ohba. Among, all of these species, $R$. rosea has been the predominant subject of phytochemical, animal and human studies (Shikov, 2014).

The root of Rhodiola rosea is mentioned in Carl Linne's Materia Medica, which recommends it as a treatment for headaches, hysteria, and as an astringent. The traditional medicinal uses of $R$. rosea have been well established, and numerous studies have been carried out in-vitro and in-vivo concerning cardio-, neuro- and hepatoprotective effects, antiviral, anti-inflammatory and antibacterial activities of $R$. rosea extracts (Panossian et al., 2010).

$R$. rosea has a long history of use as a medicinal plant in several traditional systems. Between 1748 and 1961 a catalogue of medicinal applications of $R$. rosea appeared in the scientific literature of Sweden, Norway, France, Germany, Iceland, and the Soviet Union, principally as an adaptogen with various health-promoting effects (Panossian et al., 2010).

The root of $R$. rosea is mainly wild-harvested, the main region being the Altai Mountains in southern Siberia (Galambosi, 2005). Although some attempts have been made to cultivate (e.g. Canada, Sweden, Bulgaria), there appears to be little interest for investment in large-scale cultivation. This may be due to the long-term nature of any investment (the root needs five years of growth before it can be harvested) or it may be that the costs involved make it difficult for farmers to compete on price with the wild-collected material.

However, our initial investigations suggest that due to its popularity, there is a scarcity of authentic raw material available and not enough to satisfy demand. Because of this disparity between supply (at an acceptable price) and demand, there is potential for adulteration with different species, especially Rhodiola crenulata (Hook.f. \& Thomson) H.Ohba and other Chinese species.

With a traditional history of medicinal use within Eastern Europe, Scandinavia, arctic countries, Asia and North America - Rhodiola products have become high value commodities, traded internationally. One factor contributing towards this economic growth is its use by sports men and women to help prevent fatigue and improve performance (Parisi et al., 2010). Use of $R$. rosea and other Rhodiola species is allowed by sports regulators but this use highlights a potential danger that if adulterated products are unknowingly used, the reputation of sports competitors could be adversely affected. 
Herbal products, advertised as containing the root of $R$. rosea, are widely available from retails outlets and the internet. They are generally sold as 'food supplements' and so providing they do not make any medicinal claim they can legally be placed on the market without the need of either a medicines product licence (PL) or a traditional herbal medicinal product registration (THR). However, there are several $R$. rosea products available that are registered herbal medicinal products for use in the treatment of stress-induced fatigue, exhaustion and anxiety.

Products registered under the THR scheme must demonstrate that they are safe and of acceptable quality, including adherence to good agricultural and collection practices (GACP) and good manufacturing practice (GMP).

\section{Aims and Objectives}

To select a sample of Rhodiola products, available from the internet and from retail outlets and assess their phytochemical composition.

To determine whether there are phytochemical differences between registered products and unregistered products and to assess how this impacts on the products' quality (and thus on safety as well as consumer confidence).

\section{Materials and methods}

\section{Test samples}

Approximately 40 products were obtained from different suppliers including retail outlets and products that are readily available over the Internet. The samples claimed to be consisted of crude drug material (2), bulk powder (3), hard capsule extracts (21), soft gel capsules (1), tablets (9), of Rhodiola rosea and aqueous extracts of Rhodiola crenulata (3). 37 were mono-preparations claiming to contain only Rhodiola rosea root and rhizome extracts (and excipients), and two were combined with other constituents such as vitamins, and herbal extracts. Two of the products held a traditional herbal registration (THR), but the majority were not registered products and were readily available over the Internet and from retail outlets (as food supplements). A detailed description of all investigated products is provided in the supplementary data.

\section{Solvents, reagents and chemicals}

Deuterated dimethyl sulfoxide-D6 lot no. 14F-145 and tetramethylsilane (99.9\%) lot no S47541 32108 B02 were purchased from Cambridge Isotope Laboratories, Inc., Andover, MA, USA. Ethanol absolute (99.8\%), lot no 950-0090 purchased from Merck, Germany, Methanol, lot no 982801 purchased from CarlRoth GmbH, Karlsruhe, Germany, Ethylacetate (99.5\%), lot no A0343909 purchased from Acros, New Jersey, USA, Formic acid (98+ \%) pure, lot no A0333265 purchased from Acros, New Jersey, USA. 
139 Reference standard: Salidroside, lot no BCBH4124V and rosavin, lot no 083M4725V were purchased from

140 Sigma-Aldrich Chemicals, St Luis, MO, USA.

141 'H-NMR spectroscopy

142 Preparations of standard solutions and samples

143 Approximately $50 \mathrm{mg}$ of solid samples was accurately weighed and transferred to a $1.5 \mathrm{ml}$ Eppendorf reaction 144 tube, $1 \mathrm{ml}$ of deuterated DMSO containing $0.05 \%$ tetramethylsilane were added. The mixture was mixed on a 145 rotary mixer for $60 \mathrm{~s}$, sonicated for 10 minutes at room temperature and centrifuged for 10 minutes at room 146 temperature (speed; 14,000 rpm). The reference standard solutions of salidroside, and rosavin were prepared 147 at a concentration of $1.0 \mathrm{mg} / \mathrm{ml}$ in deuterated DMSO. Seven hundred microliters of supernatant was 148 transferred to a $5 \mathrm{~mm}$ diameter NMR tube, and the samples were submitted on the same day for ${ }^{1} \mathrm{H}-\mathrm{NMR}$ 149 analysis. Sample 39 was extracted twice (S40) to act as a control for the statistical analysis.

\section{Apparatus and instrumentation}

151 NMR spectra were recorded on Bruker Avance $500 \mathrm{MHz}$ spectrometer $\left({ }^{1} \mathrm{H}, 500 \mathrm{MHz}\right)$ equipped with a QNP 152 multi-nuclear probe head with z-gradient. The topspin software version 1.3 was used for spectra acquisition and processing. The AMIX Bruker Biospin multivariate analysis software version 3.0 was used for converting spectra to an ASCII file. The numbers of scans chosen was 64 for optimum resolution of peaks, and locked at zero on the TMS peak. Principal Component Analysis (PCA) carried out using SIMCA software version 13.0.

\section{LC-MS/MS Analysis}

Preparations of standard solutions and samples

158 One mg of substance was suspended in $1 \mathrm{~mL}$ of solvent $\mathrm{A}(2.5 \%$ (v/v) MeCN, $0.5 \%$ (v/v) HCOOH in water), sonicated for 10 minutes and centrifuged at room temperature for 10 minutes at $5000 \mathrm{~g}$. Apparatus and instrumentation The LC-MS/MS data was acquired with an Agilent 1100 HPLC system which was coupled to a Bruker Daltonics

162 Esquire HCT ion-trap mass detector. $10 \mu \mathrm{L}$ of the supernatant were used as injection volume for the HPLC. The 163 chromatographic step was performed with a constant flow rate of $0.2 \mathrm{ml} / \mathrm{min}$ and a 45 minutes linear gradient 164 from solvent A to solvent B $(95 \% \mathrm{MeCN}(\mathrm{v} / \mathrm{v}), 0.5 \%(\mathrm{v} / \mathrm{v}) \mathrm{HCOOH}$ in water). For separation, a Waters Atlantis T3 column $(2.1 \times 150 \mathrm{~mm}$, Silica $3 \mu \mathrm{m})$ was used which was heated to $50^{\circ} \mathrm{C}$.

167 The ${ }^{1} \mathrm{H}-\mathrm{NMR}$ spectra were phase-corrected, baseline-corrected, and zeroed to the TMS peak. The spectra were converted to an ASCII file using AMIX software for multivariate analysis. AMIX was used to generate a number of integrated regions (buckets) of the data set. The size of buckets was $0.04 \mathrm{ppm}$. The signals of deuterated

170 DMSO, residual water, and TMS internal standard were removed before performing the statistical analysis. The 
171 data set was imported to Microsoft EXCEL, and the samples were labelled 1 to 40. The Principal Component

172 Analysis (PCA) was carried out using SIMCA software version 13.0.

173 High performance thin layer chromatography (HPTLC)

Preparations of standard solutions and samples

The extraction of plant sample was performed based on a method described by the HPTLC association for the identification of dried Rhodiola root. The reference standard solutions of salidroside and rosarin were prepared at concentration of $1.0 \mathrm{mg} / \mathrm{ml}$ in methanol. Approximately $500 \mathrm{mg}$ of solid samples were weighed individually into $10 \mathrm{ml}$ reaction tubes and $5 \mathrm{ml}$ of ethanol (99.8\%) was added. The resultant solution was shaken at $300 \mathrm{rpm}$ for 10 minutes and centrifuged for 5 minutes at $5000 \mathrm{rpm}$. The supernatant solution was transferred into individual vials, and then submitted for HPTLC analysis.

Chromatography HPTLC analysis was performed on $200.0 * 100.0$ mm silica gel 60 F 254 HPTLC glass plates (Merck, Germany).

183 Standard solutions and samples $(2 \mu \mathrm{l})$ were applied on the plate as bands $8.0 \mathrm{~mm}$ wide using CAMAG spray-on technique with Automatic TLC sampler (ATS 4) or CAMAG Linomat 5. Bands were applied to a distance of $8.0 \mathrm{~mm}$ from the lower edge of plate and $20 \mathrm{~mm}$ from the left and right edges. The space between bands was $11 \mathrm{~mm}$, and the number of tracks per-plate was 15 . The development distance was $70.0 \mathrm{~mm}$ from the lower edge of the plate using CAMAG Automatic developing chamber (ADC2). The temperature and the relative humidity within the developing chamber (ADC2) were $23^{\circ} \mathrm{C}$ and $33 \%$ respectively.

The derivatisation of plates was performed through dipping (Speed: 3, time:0) with $10 \%$ sulphuric acid in methanol reagent using CAMAG Chromatogram Immersion Device and heated to $100^{\circ} \mathrm{C}$ for 5 minutes on the

192 TLC plate heater. The plates were documented using CAMAG Visualizer under white light, UV $254 \mathrm{~nm}$, and UV $366 \mathrm{~nm}$ with visionCATS software.

\section{Other Apparatus and instrumentation}

POS-3000, Grantbio, Serial no b090250014, Cambridgeshire, England.

\section{Results and Discussion}

199 The samples were analysed by ${ }^{1} \mathrm{H}-\mathrm{NMR}$ spectroscopy coupled with SIMCA multivariate analysis software, mass spectrometry and HPTLC. assigned and compared with a Rhodiola rosea crude dried plant root supplied by a company that manufactures THR products (S35). 


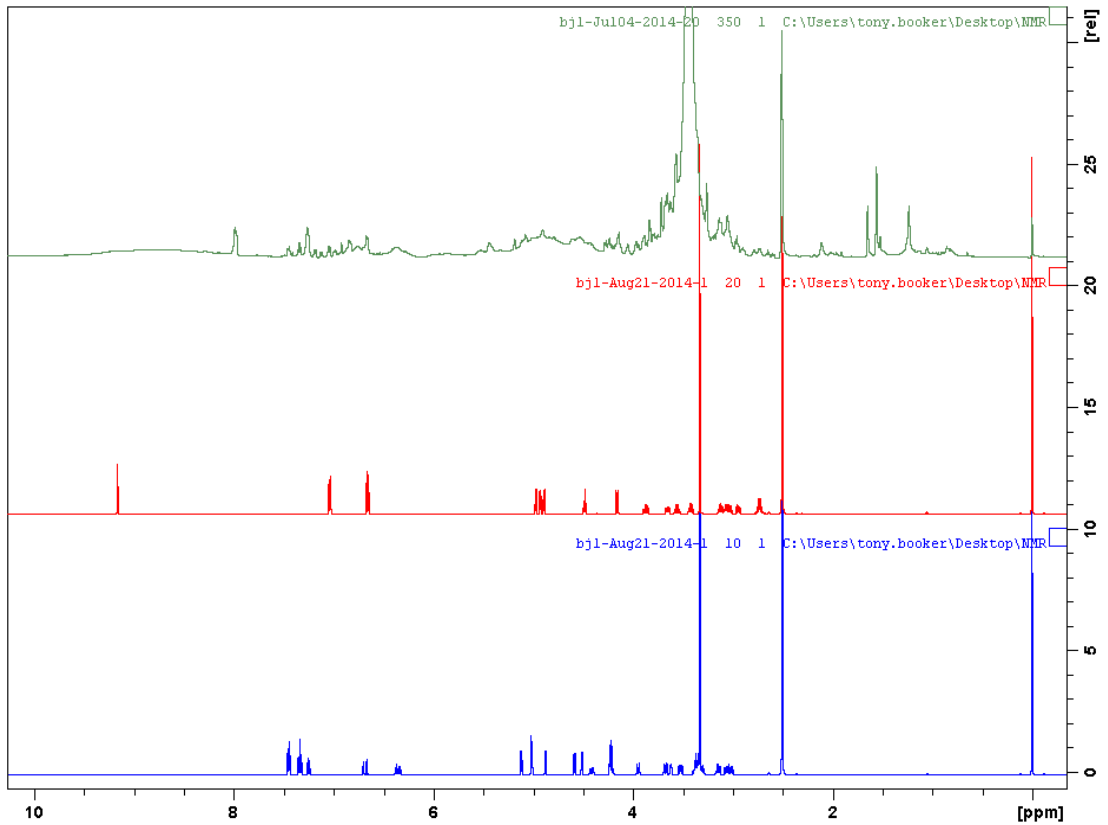

Figure $1^{1} \mathrm{H}-\mathrm{NMR}$ spectra of Rhodiola rosea crude dried plant (top) together with spectra of reference standards of salidroside (middle) and rosavin (bottom) respectively.

Because of the high concentration of glycosides, including polysaccharides and the presence of excipients in many of the Rhodiola products, it was difficult to identify peaks in the carbohydrate range ( $3-5 \mathrm{ppm})$. The resonances observed at lower chemical shifts $(0.2-3.0 \mathrm{ppm})$ are most probably attributable to the methyl groups of terpenoids (Fig. 1). However, peaks corresponding to salidroside and rosavin could be observed in the aromatic region of the spectrum (6.2-8.5 ppm) (Fig. 2). Using this method we were able to confirm the presence of both saldroside and rosavin in our samples.

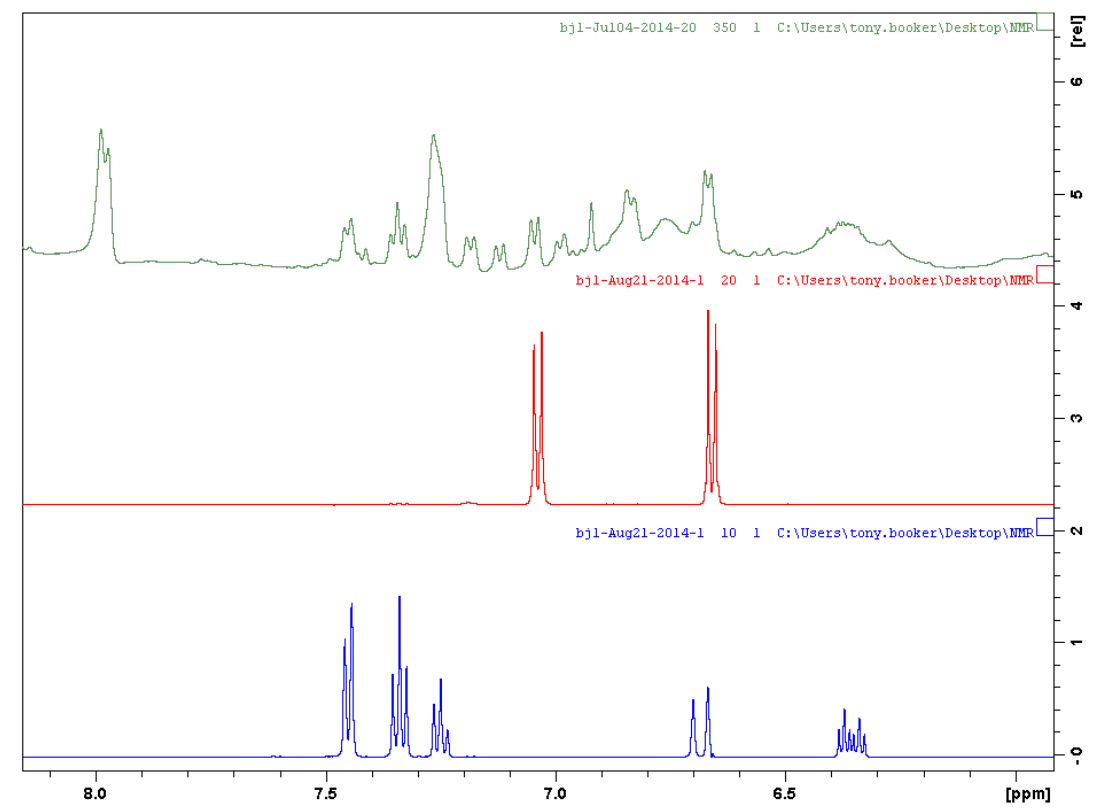

Figure $2^{1} \mathrm{H}$-NMR spectra of $R$. rosea extract (top) together with spectra of reference standards of salidroside (middle) and rosavin (bottom), respectively, focusing on aromatic region. 

$10 \mathrm{ppm}$ ). The Rhodiola products PCA appeared to indicate that although there was much variation between samples, the variation did not distinguish between the different species. The principal variation between these two species is rosavin content.

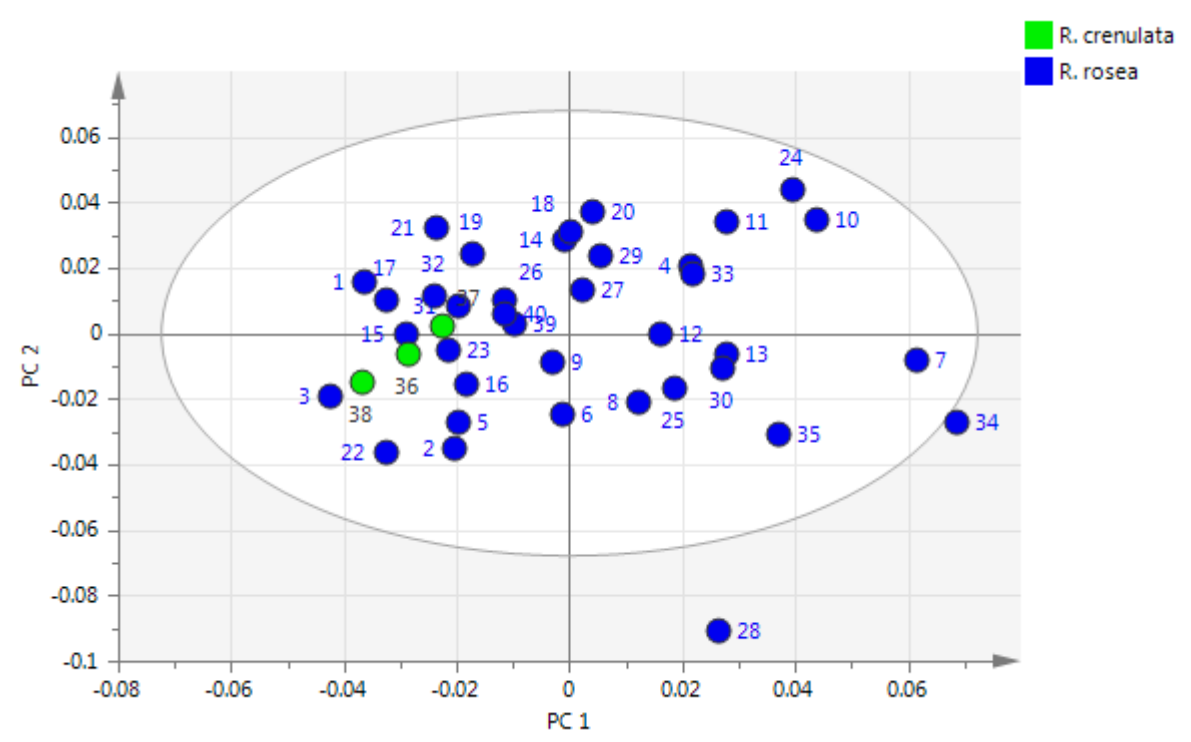

220

Figure 3 Scores plot showing the whole spectral regions of investigated Rhodiola samples

The PCA grouping in Fig. 3 may be attributable to the similarities of major metabolites, e.g. glycosidic constituents within Rhodiola products or to ingredients added (mainly excipients) to the commercial extracts. This PCA model appeared to poorly discriminate between products containing $R$. rosea extracts and those extracts containing Rhodiola crenulata or other Rhodiola species. Our assumption was that this observation was possibly due to adulteration of products by incorrect species, i.e. that a proportion of products labelled $R$. rosea actually contained an amount of $R$. crenulata or other species. In order to investigate this hypothesis, subsequent analysis was performed utilising HPTLC.

We developed an HPTLC method in order to simultaneously detect the presence of rosavin and salidroside. Importantly, $R$. rosea contains both salidroside $\left(R_{F} 0.35\right)$ and rosavin $\left(R_{F} 0.22\right)$, whereas although salidroside is present in many other Rhodiola species, rosavin is absent or in very low concentrations (Panossian 2010). The characteristic marker compound rosavin was not found in seven of the products claiming to be $R$. rosea and the overall fingerprint looked very different to that of $R$. rosea (Fig 4). Sample (S) 11 was a registered product and S20 was an expired $R$. rosea product that we included to see if the marker compounds were still visible past the expiry date. 


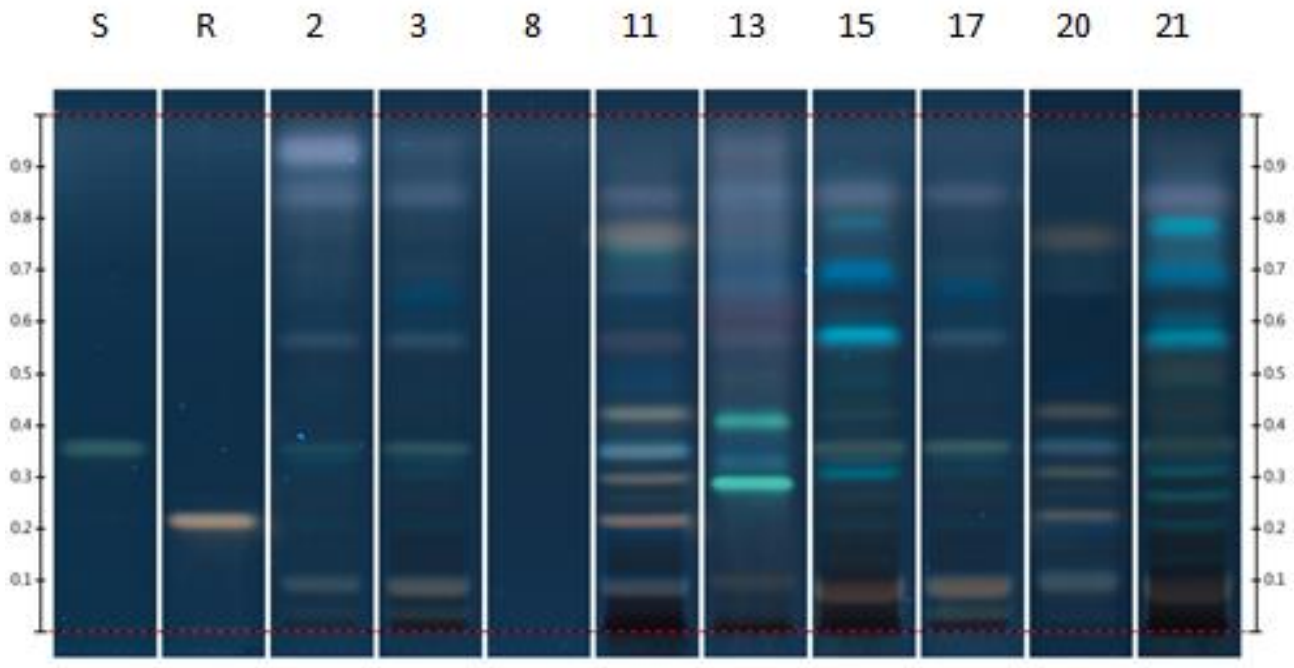

238

239 Figure 4 Comparing different Rhodiola products, Mobile phase: Ethylacetate, methanol, water, formic acid 240 (77:13:10:2). D: Sulphuric acid reagent, UV $366 \mathrm{~nm}$.

242 The HPTLC analysis revealed that S2, S3, S15, S17, and S21 samples show a fingerprint not consistent with that 243 of Rhodiola rosea and were probably adulterated with other Rhodiola species that did not contain rosavin $\left(R_{F}\right.$ $2440.2)$ but contained salidroside $\left(R_{F} 0.35\right)$ e.g. Rhodiola crenulata, Rhodiola quadrifida. Moreover, two of the 245 samples exhibited the presence of unspecified components, not related to R. rosea or Rhodiola species (S8 and 246 S13).

247 S2 displayed a strong zone at $R_{F} 0.92$, S13 displayed brightly coloured greenish zones $\left(R_{F} 0.3\right.$ and 0.4$) S 8$ 248 appeared not to contain any compounds detectable under this system.

249 After discovering that these seven samples were adulterated either with other Rhodiola species or unknown species, it was possible for us to re-label the assigned samples in the NMR - PCA.

251 Once re-labelled, the PCA displayed an improved differentiation between the $R$. rosea and Rhodiola crenulata samples (Fig. 5). Although we have re-labelled five of the products that did not contain rosavins as $R$. crenulata

253 it should be recognised that these may be adulterated with other Rhodiola species and we are currently developing methods that will identify adulterant species within mixtures. 


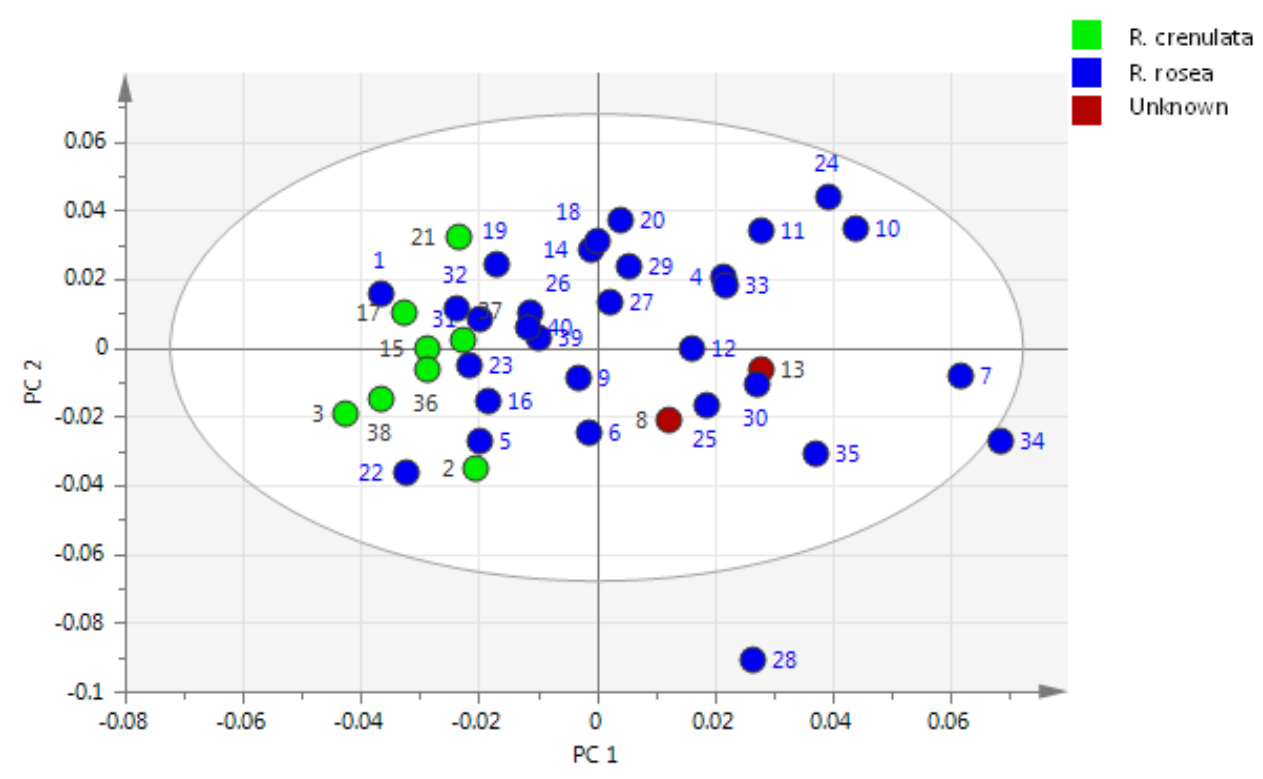

256

257

258

259

260

261

262

263

264

265

266

Figure 5 Scores plot showing the whole spectral regions of investigated Rhodiola samples after re-labelling based on HPTLC identification of the possible source species

Analysis of the raw NMR spectroscopy data for $\mathrm{S} 8$ in the aromatic region lead to the identification of signals which are characteristic for derivatives of the amino acid tryptophan. A downfield shifted signal at 10.6ppm was characteristic for an indole ring- $\mathrm{NH}$. Closer analysis of the coupling pattern for resonances between $6.5 \mathrm{ppm}$ and 7.1ppm identified a lack of a carbon bound proton at position 5 of the indole moiety. This led to the assumption, that the substance is 5-hydroxytryptophan (5-HTP). This assumption was proved by comparing NMR (Fig. 6) and LC-MS/MS spectra (Fig. 7) of a commercial sample of 5-HTP with S8. Both, the NMR and the LC-MS/MS spectra confirmed a presence of 5-HTP in S8 with the same resonances, elution times and molecular masses, respectively.

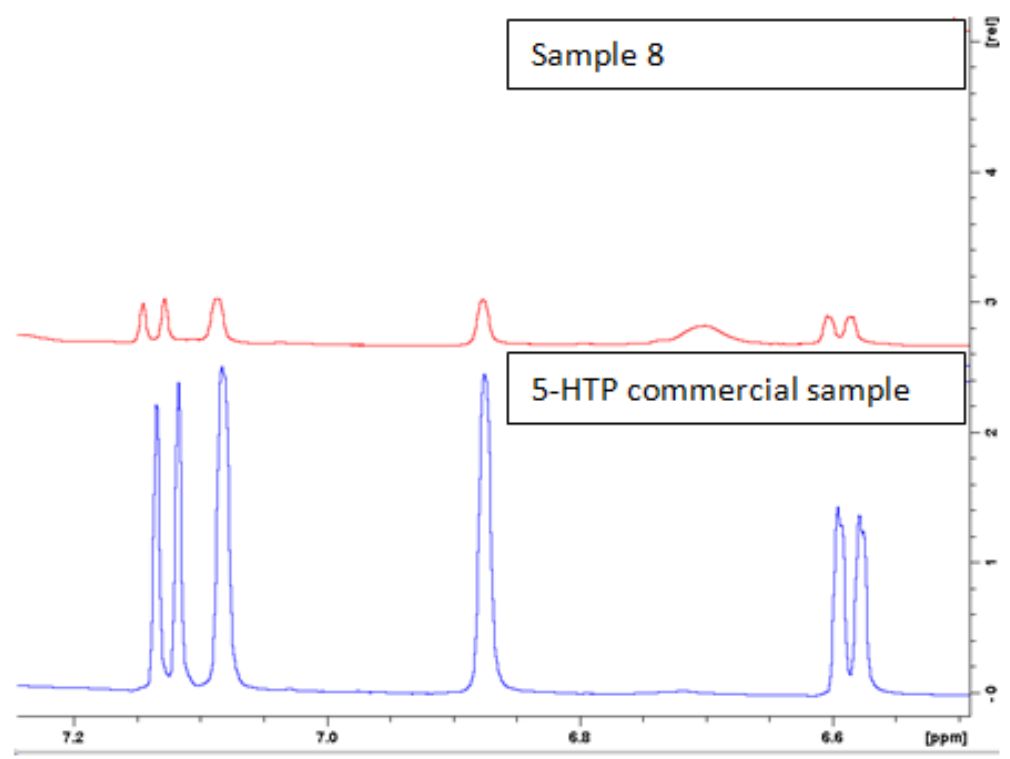



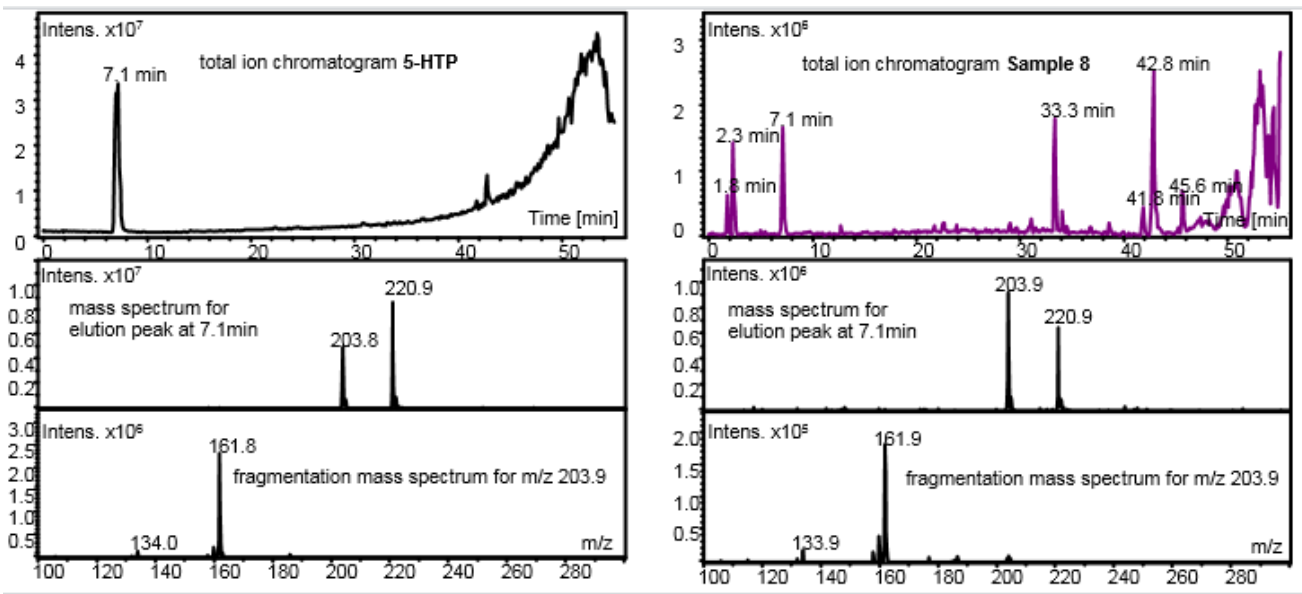

Figure 7 LC-MS/MS chromatogram with the mass spectra and fragmentations in positive and negative ion mode for 5-HTP reference standard and S8. The elution fraction at $7.1 \mathrm{~min}$ for 5-HTP with its characteristic fragmentation is also detectable for 58.

One of the problematic factors in the metabolomic analysis of commercial products is the presence of excipients. These tend to occur within the carbohydrate region and, in order to minimise the effect of any excipient, we performed a PCA exclusively on the aromatic region (7-10ppm).

In order to compare only the aqueous and hydro-ethanolic extract products, the crude raw drugs (samples 1, 34 and 35) were removed from the data set, together with the unknown products (samples 8 and 13) and a soft-gel extract (sample 28). The resulting PCA showed a better differentiation between $R$. rosea and the nonrosea species. Further investigations will determine whether these are $R$. crenulata or other adulterant Rhodiola species (Fig. 8).

It is observed that the products that are highest in rosavin are in the bottom right quadrant. There is a group of seven products that are higher in rosavin content than other products, including the 2 THR products (11 and 33). However, there are some $R$. rosea products that appear to have a very low rosavin content; in fact the PCA places these more closely to the group we have labelled $R$. crenulata products (samples 5 and 6 ). The likely reason for this is that these products contain a mixture of $R$. rosea and $R$. crenulata or other adulterant species. Through closer examination of this PCA we can infer that over $80 \%$ of products contain less rosavin than the THR products. 


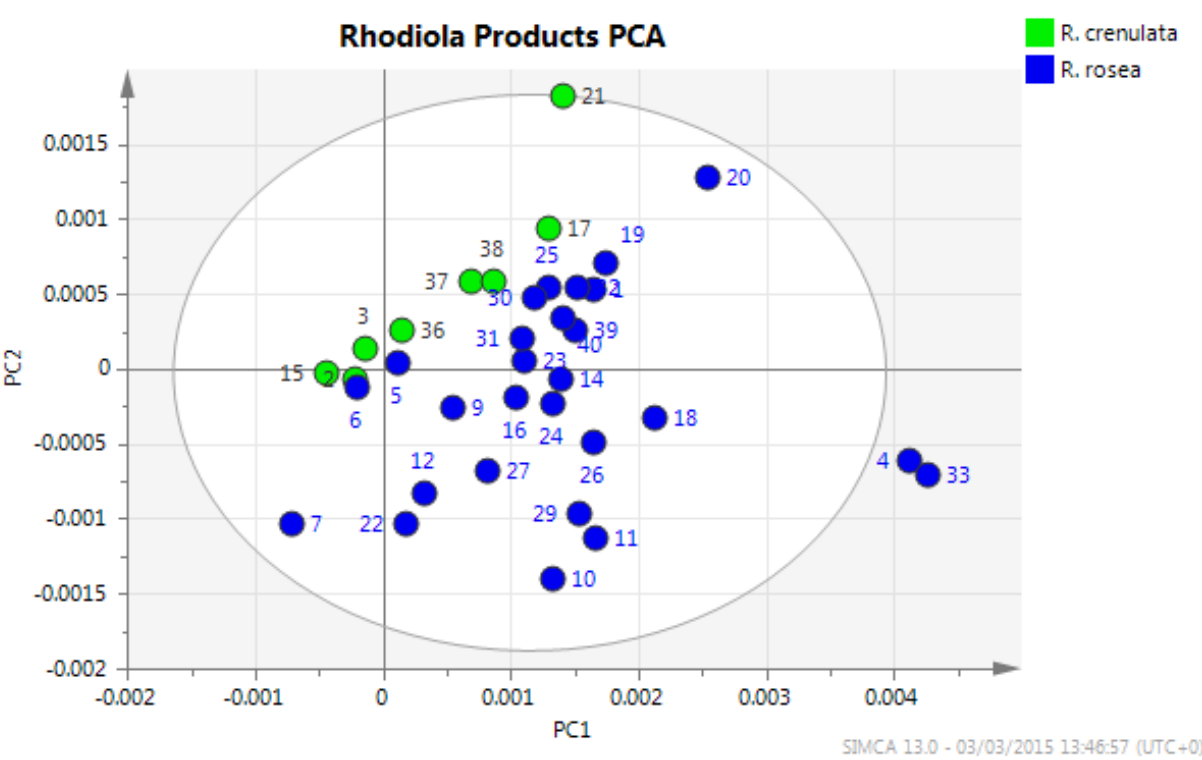

Figure 8 Scores plot showing PCA of Rhodiola products (7-10 ppm chemical shift). The lower ellipse indicates products that are higher in rosavin content.

Table 1 Label claims versus actual findings for Rhodiola products

\begin{tabular}{|c|c|c|}
\hline Sample no. & & Findings \\
\hline 2 & Rhodiola rosea sourced in China & $\begin{array}{l}\text { Not } R \text {. rosea, probably other rhodiola } \\
\text { species e.g. } R \text {. crenulata }\end{array}$ \\
\hline 3 & Whole dried root of Rhodiola rosea & $\begin{array}{l}\text { Not } R \text {. rosea, probably other rhodiola } \\
\text { species e.g. } R \text {. crenulata }\end{array}$ \\
\hline 8 & $\begin{array}{l}\text { Rhodiola rosea extract } 2000 \text { mg, } \\
\text { wild-sourced from Siberia }\end{array}$ & $\begin{array}{l}\text { Not } R \text {. rosea or any other R. species. } \\
\text { Determined as } 5 \text {-HTP and excipients }\end{array}$ \\
\hline 13 & Rhodiola rosea root $1000 \mathrm{mg}$ & $\begin{array}{l}\text { Probably not rhodiola species, appears } \\
\text { adulterated }\end{array}$ \\
\hline 15 & $\begin{array}{l}\text { Rhodiola rosea plus multivitamins } \\
\text { standardised to contain } 3 \% \text { rosavins } \\
\text { and } 1 \% \text { salidroside }\end{array}$ & $\begin{array}{l}\text { Not } R \text {. rosea, probably other rhodiola } \\
\text { species e.g. } R \text {. crenulata }\end{array}$ \\
\hline 17 & $\begin{array}{l}\text { Rhodiola rosea standardised to } \\
\text { contain } 1 \% \text { salidroside }\end{array}$ & $\begin{array}{l}\text { Not } R \text {. rosea, probably other rhodiola } \\
\text { species e.g. } R \text {. crenulata }\end{array}$ \\
\hline 21 & $\begin{array}{l}\text { Rhodiola rosea standardised to } \\
\text { contain } 1 \% \text { salidroside }\end{array}$ & $\begin{array}{l}\text { Not } R \text {. rosea, probably other rhodiopla } \\
\text { species e.g. } R \text {. crenulata. Probable high } \\
\text { sugar content }\end{array}$ \\
\hline
\end{tabular}


The safety and quality of herbal medicines available to European consumers has been a key issue for medicines regulators. The introduction of the Traditional Herbal Medicinal Products Directive (THMPD) and herbal registration has provided a means whereby consumers can access a wide variety of popular herbal medicines of assured quality and with a well-researched safety profile. However, there are still a large number of medicines left un-regulated and widely available, particularly from internet sources. Economically lucrative products, including $R$. rosea products, are likely candidates for adulteration, especially when the raw plant material is in short supply. Moreover, because $R$. rosea products are popular with sports persons, there is an added danger that they may be adulterated with performance enhancing stimulants.

Using a methodology previously developed by our group (Booker et al., 2014), we investigated the utilisation of ${ }^{1} \mathrm{H}-\mathrm{NMR}$ spectroscopy coupled with multivariate analysis software and HPTLC to ascertain the identity and composition of $R$. rosea value chain products. Both techniques provide different and complementary data, which we used to discriminate between the wide-variety of sampled finished products. Further investigations will focus on the crude drug material and aim to establish a robust method for identifying mixtures of different species within products.

${ }^{1} \mathrm{H}-\mathrm{NMR}$ spectroscopy coupled with multivariate analysis software enabled us to group the Rhodiola products based on similarities between the products. Although chemometric data analysis can be undertaken automatically with the requisite software packages, it requires an in-depth understanding as to what each set of signals in the given spectra represents phytochemically, so that the identification of key constituents within

314 a product can be assigned logically. In this set of samples (mainly extracts) there were a lot of overlapping peaks present, particularly in the carbohydrate region, probably produced by a combination of intrinsic glycosidic material and excipients. This made interpretation of the data difficult for individual compounds and suggested that a more separation technique, e.g. HPTLC should be used. The HPTLC analysis provided detailed qualitative data for the determination of the marker compounds, and allowed to make visual comparisons between different products relatively easily. Because HPTLC allows us to be selective regarding the groups of compounds we want to analyse, we can make comparisons between samples based on the composition of the main marker compounds (salidroside and rosavin). Pharmacopoeia. However, $R$. rosea is the species of main economic interest. The difference in price between 324 the two species, with $R$. crenulata being cheaper for the foreign buyer, and the relative ease of availability in 325 China of $R$. crenulata, may be a reason behind the adulteration of $R$. rosea products with $R$. crenulata and possibly other Rhodiola species. While there is - at this stage - no evidence for potential risks in using Rhodiola crenulata, apart from a possible lack of effectiveness, other examples of phytomedicines highlight the risk of adulteration with a species of the same genus ( $L i$ and $\mathrm{Yu}, 2006$ ). 
In this study, the characteristic marker compound (rosavin) was not detected in twenty three percent of unregistered products that claimed to contain $R$. rose. Two of these products were adulterated with material not from the genus Rhodiola i.e. that did not contain either rosavin or salidroside and one of these was positively identified as 5-hydroxytryptophan, an amino acid commonly used as an anti-depressant or an aid for weight loss. It is unclear whether this is a deliberate or accidental adulteration since attempts to clarify this with the companies involved have been unsuccessful. However, whether deliberate or not, these findings show that there is a monumental failing in the quality systems of the companies involved. Moreover, there were variations in the amount of marker compounds contained in Rhodiola products. This was supported by the PCA data which indicated that approximately $80 \%$ of products contained lower amounts of rosavin than the THR products. Thus, this may indicate that there are common but qualitative different species of Rhodiola substituted or used as admixtures in $R$. rosea labelled products.

While adulteration by other Rhodiola species, including Rhodiola crenulata presents one particular problem, adulteration with unknown material is potentially even more worrying. Future investigations will focus on the isolation and identification of these compounds to determine whether there are other plant species or synthetic compounds (including stimulants) and how different value chains are affected.

\section{Conclusions}

Approximately one quarter of unregistered Rhodiola products were adulterated and did not conform to their label specification. Approximately $80 \%$ of products were of poorer quality than the THR products as regards the rosavin content. This indicates that there are major breakdowns in the quality systems employed along the various stages of Rhodiola value chains. Buying unregistered products, particularly from the internet, presents a clear risk. There is no practical way for the general public to differentiate un-registered genuine products from adulterated products. Products registered under the THMPD were confirmed to contain authentic RR. investigate how and why such adulterations can happen. This research also calls for more training and for raising awareness of the relevant stakeholders.

Conflicts of interest

We wish to draw the attention of the Editor to the following facts which may be considered as potential conflicts of interest and to significant financial contributions to this work. Co. KG, Germany. he identified 5-hydroxytryptophan in one of the samples and conducted the LC-MS/MS-experiments for the 
366 Ali, Z., Fronczek, F.R., Khan, I.A. (2008). Phenylalkanoids and Monoterpene Analogues from the Roots of 367 Rhodiola rosea. Planta Medica, 74, 178-181.

368 Booker, A., Frommenwiler, D., Johnston, D., Umealajekkwu, C., Reich, E., Heinrich, M. (2014). Chemical variability along the value chains of turmeric (Curcuma longa): A comparison of nuclear magnetic resonance spectroscopy and high performance thin layer chromatography. Journal of Ethnopharmacology, 152, $292-301$. Brown, R.P., Gerbarg, P.L., Ramazanov, Z. (2002). Rhodiola rosea: A phytomedicinal overview. HerbalGram (American Botanical Council), 56, 40-52

373 Galambosi, B. (2005). Rhodiola rosea L., from Wild Collection to Field Production. Medicinal Plant Conservation, 11, 31-35.

Ganzera, M., Yayla, Y., Khan, I.A. (2001). Analysis of the marker compounds of Rhodiola rosea L., (Golden root) by reversed phase high performance liquid chromatography. Chemical Pharmaceutical. Bulletin, 49, 465-467. Kim, H.K., Choi, Y.H., Verpoorte, R. (2010). NMR-based metabolic analysis of plants. Nature Protocols, 5(3), 378 536-549. Li JX, Yu ZY. (2006) Cimicifugae rhizoma: from origins, bioactive constituents to clinical outcomes. Current Medical Chemistry,13(24):2927-51.

Ma, Y.C., Wang, X.Q., Hou, F., Ma, J., Luo, M., Lu, S., Jin, P., Terevsky, N., Sautreau, A., Gorecki, D. (2011). Rapid resolution liquid chromatography (RRLC) analysis for quality control of Rhodiola rosea roots and commercial standardized products. Natural Product Communication, 6, 645-650.

Ndjoko loset, K., Nyberg, N.T., Van Dierman, D., Malnoe, P., Hostettmann, K., Shikov, A.N., Jaroszewski, J.W. (2011). Metabolic profiling of Rhodiola rosea rhizomes by H NMR spectroscopy. Phytochemical Analysis, 22, 158-165.

Parisi, A., Tranchita, E., Duranti, G., Ciminelli, E., Quaranta, F., Ceci, R., Cerulli, C., Borrione, P., Sabatini, S. (2010). Effects of chronic Rhodiola Rosea supplementation on sport performance and antioxidant capacity in trained male: preliminary results. Journal of Sports Medicine and Physical Fitness, Mar; 50(1), 57-63. Panossian, A., Wikman, G., \& Sarris, J. (2010). Rosenroot (Rhodiola rosea): Traditional use, chemical composition, pharmacology and clinical efficacy. Phytomedicine, 17(7), 481-493.

Peschel, W., Prieto, J.M., Karkour, C., Williamson, E.M. (2013). Effect of provenance, plant part and processing on extract profiles from cultivated European Rhodiola rosea L. for medicinal use. Phytochemistry, 86, 92-102.

Shikov, A.N., Pozharitskaya, O.N., Makarov, V.G., Wagner, H., Verpoorte, R., Heinrich, M. (2014). Medicinal Plants of the Russian Pharmacopoeia; their history and applications. Journal of Ethnopharmacology, 154, 481536.

Yousef, G.G., Grace, M.H., Cheng, D.M., Belolipov, I.V., Raskin, I., Lila, M.A. (2006). Comparative phytochemical characterization of three Rhodiola species. Phytochemistry, 67, 2380-2391. 